\title{
Tuning intermolecular interactions in di-octyl substituted polyfluorene via hydrostatic pressure
}

\author{
K. Paudel, M. Chandrasekhar, and S. Guha* \\ Department of Physics and Astronomy, \\ University of Missouri-Columbia, Missouri 65211
}

(Dated: November 20, 2018)

\begin{abstract}
Polyfluorenes (PFs) represent a unique class of poly para-phenylene based blue-emitting polymers with intriguing structure-property relationships. Slight variations in the choice of functionalizing side chains result in dramatic differences in the inter- and intra-chain structures in PFs. We present photoluminescence (PL) and Raman scattering studies of bulk samples and thin films of dioctylsubstituted PF (PF8) under hydrostatic pressure. The bulk sample was further thermally annealed at $1.9 \mathrm{GPa}$. The PL vibronics of the as-is sample red-shift at an average rate of $26 \mathrm{meV} / \mathrm{GPa}$. The thermally annealed sample is characterized by at least two phase transitions at $1.1 \mathrm{GPa}$ and 4.2 GPa, each of which has a different pressure coefficient for PL vibronics. The Huang-Rhys factor, a measure of the electron-phonon interaction, is found to increase with increasing pressures signaling a higher geometric relaxation of the electronic states. The Raman peaks harden with increasing pressures; the intra-ring C-C stretch frequency at $1600 \mathrm{~cm}^{-1}$ has a pressure coefficient of $7.2 \mathrm{~cm}^{-1} / \mathrm{GPa}$ and exhibits asymmetric line shapes at higher pressures, characteristic of a strong electron-phonon interaction. The optical properties of PF8 under high pressure are further contrasted with those of a branched side chain substituted PF.
\end{abstract}

PACS numbers: $61.50 . \mathrm{Ks}, 71.20 . \mathrm{Rv}, 78.30 . \mathrm{Jw}$ 


\section{INTRODUCTION}

Blue-emitting polymers continue to attract a lot of attention for display applications. Among them polyfluorenes (PFs), which belong to a class of poly para-phenylene (PPP) system, are extremely attractive not only from the technological perspective due to their high photoluminescence quantum yield, but also from the viewpoint of serving as model systems for understanding fundamental photophysical phenomena owing to their rich phase morphology. Molecular level attributes such as local chain structure and side chain conformations in these systems strongly impact their transport and device characteristics. Slight variations in the choice of functionalizing side chains result in dramatic differences in the inter- and intra-chain structures in PFs. Highlighting these differences are two prototypical PFs, poly (9,9-(di-octyl) fluorene) (PF8) and poly (9,9-(di ethyl-hexyl) fluorene) (PF2/6). Side chain substitution gives rise to a rich array of mesomorphic behavior with the appearance of a nematic liquid crystalline $\left(n\right.$-LC) phase at higher temperatures. $\frac{1}{n}$ In the last decade many research groups have focused on structural studies of PFs using x-ray scattering, $, 2,3,4$

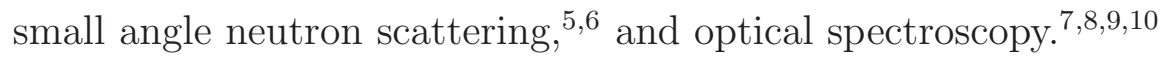

PFs are characterized by 3-D crystalline phases as well as conformational phases or chromophores, where the latter depends on the structure of the individual chains. PF2/6 has a limited number of conformational isomers that form a fivefold helix $(5 / 2$ or $5 / 1) \underline{11}$ These helices in turn self-organize into three chain unit cells resulting in a well ordered semicrystalline hexagonal phase with coherence lengths exceeding $50 \mathrm{~nm} \underline{\underline{4}}^{\text {Thus, }}$ the optical properties of $\mathrm{PF} 2 / 6$ are relatively insensitive to the exact crystallographic state, thermal history, or molecular weight. PF8, on the other hand, has at least three conformational isomers that depend explicitly upon the torsional angle between the adjacent monomers. ${ }^{12}$ In addition to these isomers, structural studies of PF8 have identified many crystalline phases $\underline{13}$

Recent studies have identified three different conformational isomers in terms of the local PF backbone torsional angle: $\mathrm{C}_{\alpha}$ conformer with a torsional angle of $135^{\circ}, \mathrm{C}_{\gamma}$ with an average torsional angle of $155^{\circ}$, and the almost planar conformer, $\mathrm{C}_{\beta}$, has an average torsional angle

of $165^{\circ} \stackrel{12}{=}$ The $\mathrm{C}_{\beta}$ conformer, conventionally knows as the $\beta$ phase has a separate long

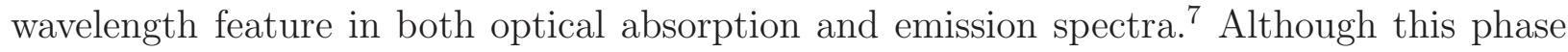
appears as a minority constituent, it dominates the optical emission. Our recent work has shown that the morphology adopted by the side chains of PF8 is closely linked to the 
structure of the molecular backbone $\stackrel{14}{15}$ For example, the $\beta$ phase backbone is stabilized when the side chains in PF8 adopt an anti-gauche-gauche conformation, as shown in the inset of Fig. 1. The rich phase morphology of PF8 is also seen in thin films of its model oligomers. $\underline{16}$

Hydrostatic pressure allows a study of materials in a region of phase space not accessible by chemical techniques. Application of hydrostatic pressure allows tuning of both intermolecular and intramolecular interactions in polymer chains without changes in the chemical make-up. $\stackrel{17}{ }$ The photophysical studies of conjugated materials under high pressure indicate that enhanced intermolecular interaction produces an increased degree of conjugation, increased exciton trapping in organic polycrystalline molecules, $\frac{18}{2}$ redshift and broadening of the photoluminescence,,$\frac{19}{20,21,22}$ enhancement of excited state dynamics at polymerpolymer heterojunctions, ${ }^{23}$ destabilization of localized states as in methylated ladder-type PPP (MeLPPP),$\stackrel{24}{\Perp}$ and changes in the ring torsion motion as in non-planar para hexaphenyl (PHP) and other oligophenyls. ${ }^{25}, 26$ The pressure dependent optical studies in PHP and other oligophenyls further reveal the individual contributions of the intermolecular and intramolecular interactions. $\underline{25}$

Optical studies in PFs under pressure allow tuning of the rich phase space and its impact on electron-phonon interactions. Our previous work on PF2/6 under pressure shows dramatic changes in the PL spectrum that mainly originates from defect and aggregate states. ${ }^{27}$ The Raman peaks shift to higher energies exhibiting unexpected antiresonance line shapes at higher pressures. Although the Raman spectra show very similar behavior for PF8 and PF2/6 under pressure, indicating similar anharmonic interactions, the emission properties of the two materials are vastly different under pressure. These differences arise as a result of their unique backbone conformations: PF2/6 due to its helical backbone conformation shows a higher degree of overlap of the electronic wave-function, while PF8 also shows some excimeric emission but the changes in its PL spectrum are much more gradual compared to PF2/6. Due to the various conformational isomers, PF8 shows planarization of the backbone at relatively low pressures when pressure is applied to the more non-planar conformation, which is absent in $\mathrm{PF} 2 / 6$. 


\section{EXPERIMENTAL DETAILS}

The PF8 sample was obtained from American Dye Source (BE-129) and loaded as-is

in the pressure cell. For thin film studies under pressure, a solution of PF8 (in toluene) was drop-casted on the bottom surface of the diamond. The sticking coefficient of PF films is quite poor on diamond and therefore they experience a hydrostatic environment. The pressure studies were conducted in a Merrill-Bassett-type diamond anvil cell (DAC) with cryogenically loaded argon as the pressure medium. Pressure was measured using the luminescence of a ruby chip located in the pressure chamber. The photoluminescence (PL) spectra were excited using the $325 \mathrm{~nm}$ line of a HeCd laser or the $351 \mathrm{~nm}$ line of an $\mathrm{Ar}$ ion laser. The luminescence excitation was analyzed with an Ocean Optics 2000 spectrometer with 25-micron slits. Raman spectra were collected using an Invia Renishaw spectrometer attached to a confocal microscope with a $\times 50$ long working distance objective and the 785 $\mathrm{nm}$ line of a diode laser as the excitation wavelength. Typical laser power was a few $\mathrm{mW}$ on the sample. The bulk sample under pressure was also thermally cycled at room temperature (RT) from its $n$-LC phase at $160^{\circ} \mathrm{C}$, using similar thermal steps as in previous ambient pressure studies. 14

\section{STEADY-STATE PHOTOLUMINESCENCE}

The PL studies were carried out from two samples of PF8 loaded in DACs, one from a thin film cast from toluene and the other from the bulk sample. Both the film and bulk samples showed a signature of the $\beta$ phase before loading in the DAC. The bulk sample was thermally cycled at $1.9 \mathrm{GPa}$ by heating it to $160^{\circ} \mathrm{C}$ and bringing it slowly back to RT. Due to the higher self-absorption in the bulk sample, the high energy vibronic peaks are not very well resolved. Vibronic progressions are clearly seen in thin films of PF8, indicating a coupling of the backbone carbon-carbon stretch vibration to the electronic transitions. The transition highest in energy is the 0-0 transition, which takes place between the zeroth vibronic level in the excited state and the zeroth vibronic level in the ground state. The $0-1$ energy involves the creation of one phonon.

The peak positions of the PL vibronics in PF8 are good indicators of the nature of chain conformations; under ambient conditions the $\beta$ conformer is identified by a $0-0$ transition 




FIG. 1: PL spectra of PF8 film at selected pressures measured at room temperature. The inset shows a PF monomer with di-octyl side chains in an anti-gauche-gauche conformation.

which is almost $\sim 100 \mathrm{meV}$ red shifted compared to the $\alpha$ conformer ${ }^{28}$ Figure 1 shows the PL spectra from the thin film sample at selected values of pressure. The $2.83 \mathrm{eV}$ 0-0 emission at ambient pressure indicates the presence of the $\beta$ phase. The Raman spectra from the film also indicate the presence of this phase, as shown in Section IV. The PL vibronics are seen to red shift upon enhanced pressures. The higher vibronic peaks smear out beyond 5.0 GPa. The PL spectra in Fig. 1 were measured while pressure was being increased. The hysteresis is small; upon decreasing the pressure the PL spectra were almost identical to the ones shown.

Figure 2 compares the PL spectra of the as-is bulk sample before and after it was thermally cycled. The thermal cycling (TC) was carried out at $1.9 \mathrm{GPa}$, after which the PL spectra were measured upon increasing the pressure till $6.5 \mathrm{GPa}$, and then decreasing it down to 0.3 GPa. The ambient pressure 0-0 PL vibronic peak blue shifts by almost $70 \mathrm{meV}$ after TC. This is attributed to a change in the crystalline phase, discussed in greater detail in the next section. This crystallization is seen even under pressure. The PL spectra further broaden after TC. 


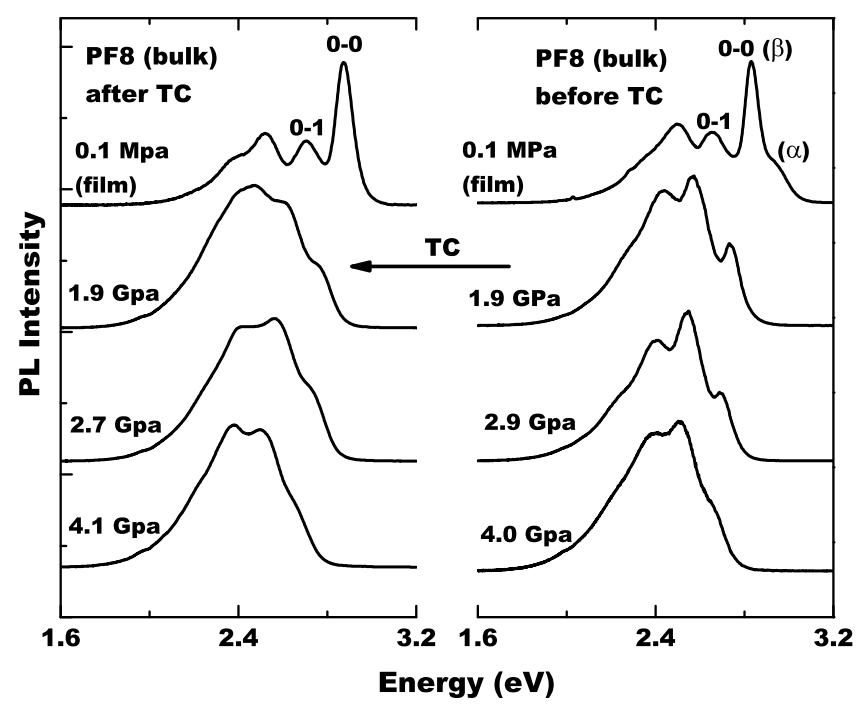

FIG. 2: The right panel shows the PL spectra of the bulk sample at selected values of pressure measured at room temperature. The left panel shows the PL spectra of the same sample after undergoing thermal cycling. The 0.1 MPa data is from a thin film sample.

\section{A. Discussion of PL results}

The complexity in deciphering the optical spectra of PF8 arises from the fact that the polymer must be simultaneously assessed in terms of its crystallographic phase and the distribution of conformational isomers. PF8 cast from various solvents appear in a metastable structure at RT $\stackrel{14}{\underline{n}}$ Cooling from $n$-LC phase yields the $\alpha$ and $\alpha^{\prime}$ crystalline phase. X-ray diffraction (XRD) studies show the $\alpha^{\prime}$ crystalline polymorph to be similar to the $\alpha$ phase and, in addition, it is exceptionally well oriented with respect to the surface normal in both ultrathin and moderately thick films. $\stackrel{2}{=}$ The progression to the $\alpha$ crystalline phase is extremely variable; PF8 films cast from various solvents are marked by an intermediate $M$ phase at RT when cooled quickly. This phase is intermediate to $\alpha$ and the $\alpha^{\prime}$, and presumably corresponds to the $\mathrm{C}_{\gamma}$ type family. A detailed Raman scattering study of PF8 as a function of thermal cycling showed that heating the polymer to $130{ }^{\circ} \mathrm{C}$ reduces the $\mathrm{C}_{\beta}$ conformer and enhances the fraction of the $\mathrm{C}_{\alpha}$ conformer $\stackrel{14}{*}$ Upon cooling the polymer back to RT from the $n$-LC phase, a higher fraction of the $\mathrm{C}_{\gamma}$ conformer is seen.

Both the film and the bulk samples of PF8 in this work were initially characterized by the $C_{\beta}$ conformer. The red-shift of the $\mathrm{PL}$ vibronics and the spectra as a whole, as seen in Figs. 1 and 2, are common to almost all conjugated polymers and molecules indicating 
a higher degree of effective conjugation. This arises due to the higher overlap between the $\pi$-electron wavefunctions. The overall PL spectrum of PF8 were fitted with 4 Gaussian peaks, three of them originating from the 0-0, 0-1, 0-2 vibronics, and a low energy defect emission. Beyond $3 \mathrm{GPa}$ the PL spectra need to be fit with an additional low energy peak at $2.0 \mathrm{eV}$, as shown for the 5.6 GPa data in Fig. 1. Most probably this peak arises from an excimer-type emission.

When PF8 is thermally cycled by cooling the sample slowly from its $n$-LC phase, it changes to an overall three-dimensional $M$ crystalline phase that precludes the $C_{\beta}$ conformer ${ }^{15}$ The resulting disordered $C_{\alpha}$ conformer, which has a non-planar backbone conformation, shows a blue-shifted $0-0$ peak at $2.9 \mathrm{eV}$ (ambient pressure data in Fig. 2). Upon thermally cycling our PF8 sample at $1.9 \mathrm{GPa}$ (indicated by the arrow in Fig. 2) the overall spectrum and the individual vibronics broaden. At this pressure the difference between the $\mathrm{PL}$ vibronic energies between the thermally cycled and the as-is sample is almost $40 \mathrm{meV}$, similar to the trend seen in the ambient pressure data. These results imply that even at 1.9 GPa the nature of the crystalline phase after TC is similar to thermal cycling at ambient pressure.

The peak positions of the 0-0 and the 0-1 PL vibronics are plotted in Fig. 3 for the film, and bulk sample before and after TC. The open symbols depict the 0-0 PL peak positions, and the filled symbols represent the 0-1 peak positions. The star symbols (filled and open) are the PL peak positions from the as-is thin film sample that did not undergo any TC. The pressure coefficients of the 0-0 and 0-1 PL vibronic peaks are listed in Table I. The pressure coefficient of the bulk (before TC) and the film samples are almost the same and yield an average pressure coefficient of $(\sim-26 \mathrm{meV} / \mathrm{GPa})$. The bulk sample after TC in contrast, shows three distinct regions with different pressure coefficients. This is more pronounced for the 0-1 PL peak as shown by a linear fit of these regions (solid black line). The data suggests distinct phase changes at $1.1 \mathrm{GPa}$ and $4.2 \mathrm{GPa}$. Beyond 4.2 GPa the thermally cycled bulk sample shows a very slow red-shift of the PL energies. For comparison, the pressure coefficient of the PL energies from a PF2/6 film are also shown 27 They are similar to as-is bulk PF8.

It is worth pointing out that in oligophenyls, where a single bond connects two phenyl rings, a sharp change in the pressure coefficient is observed in the $\mathrm{PL}$ vibronics around $\sim 1.5$ GPa; below 1.5 GPa the pressure coefficient of the PL energies is higher ${ }^{25}$ Such changes in 
oligophneyls have been attributed to planarization of the molecule. The repulsion between the ortho-hydrogen atoms leads to a torsion of neighboring phenyl rings with respect to the single bond connecting them. First principles calculation of a PPP chain predicts a decrease in the bandgap energy by almost $1 \mathrm{eV}$ when the torsional angle between the phenyl rings changes from $50^{\circ}$ to $0^{\circ} . \underline{31}$ Bulk $\mathrm{PF} 8$ before $\mathrm{TC}$ behaves similar to $\mathrm{PF} 2 / 6^{27}$ with no discernable changes in the pressure coefficients as a function of pressure. Upon TC, when the polymer crystallizes, the $\mathrm{C}_{\beta}$ conformer is eliminated and the polymer has a high fraction of $C_{\alpha}$ or $C_{\gamma}$-type chromophores. It is likely that at $1.1 \mathrm{GPa}$, pressure induces a planarization between the monomer units driving the system more towards the $\mathrm{C}_{\beta}$ conformation. Our Raman scattering studies presented in Section IV point in the same direction.

The 4.2 GPa discontinuity is not yet well understood. From our data it is clear that the sample is less compressible beyond $4.2 \mathrm{GPa}$ in TC samples. As of now the only XRD data under pressure that exist for the PF family (polymer/oligomer) is for crystalline fluorene, where a reversible phase transition is seen at $3.6 \mathrm{GPa} . \stackrel{29}{2}$ This transition corresponds to a $\pi$ stacking molecular arrangement with an increase in the bulk modulus (inverse of the compressibility). The crystal structure of PF8 is quite different from fluorene itself but the transition at $4.2 \mathrm{GPa}$ in our data may be similar to fluorene with an enhanced $\pi$ stacking geometry, which would induce a stronger inetrchain interaction. Additionally, theoretical calculations involving 3-D interactions of PPP chains show that an enhanced interchain interaction results in a broadening of the absorption/PL with a very small red-shift of the band gap. 24 Our experimental data shows a large increase in the PL linewidth of the TC sample beyond $5 \mathrm{GPa}$ (Fig. 4), pointing to the direction that at these pressures the predominant effect of hydrostatic pressure is an enhanced interchain interaction.

The PL linewdith shows differences between the as-is bulk and annealed samples. The full width at half maximum (FWHM) of the 0-1 PL vibronic is plotted in Fig. 4; the 0-0 peak also shows a similar behavior. The as-is sample clearly shows a PL broadening with increasing pressures. PL broadening as a function of pressure sheds light on the nature of intermolecular interactions and originates from the differences in compressibility of the ground and excited electronic states in configuration coordinate space..$^{30}$ In amorphous polymers such as MeLPPP, a similar broadening of the PL vibronics under pressure has been observed, $\stackrel{17}{,}$ reflecting a strong interaction perpendicular to the chain axis. The PF8 sample that was thermally cycled, on the other hand, shows a very different dependence; on an 
TABLE I: Pressure coefficients for backbone PL emission peaks in PF8 bulk (before and after thermal cycling) and film for the 0-0 and 0-1 PL peaks. The bulk sample after TC has been fitted in three regions. The pressure coefficients are determined by a linear fit to the PL energy positions versus pressure given by $E(P)=E(0)+C P$. The last row shows the pressure coefficients of the $\mathrm{PL}$ vibronic energies in PF2/6.

\begin{tabular}{ccc}
\hline \hline Sample & $C_{0-0}(\mathrm{meV} / \mathrm{GPa})$ & $C_{0-1}(\mathrm{meV} / \mathrm{GPa})$ \\
\hline PF8 bulk (before TC) & $-32.8 \pm 1.8$ & $-26.1 \pm 1.2$ \\
PF8 film (before TC) & $-25.3 \pm 0.9$ & $-21.6 \pm 1.4$ \\
PF8 bulk (after TC) & $-64.9 \pm 6.5$ & $-90.8 \pm 13.6$ \\
& $-48.3 \pm 3.6$ & $-42.6 \pm 1.5$ \\
& $-14.6 \pm 5.3$ & $-4 \pm 1.0$ \\
PF2 $/ 6$ film $^{a}$ & $-40 \pm 1.0$ & $-28 \pm 1.0$
\end{tabular}

\footnotetext{
$\overline{{ }^{a} \text { Ref. }[27}$
}

average the PL vibronics do not show any broadening till $6 \mathrm{GPa}$, beyond which a large increase in the FWHM is observed. No changes in the PL linewidths have been observed in polycrystalline molecules such as PHP 17 Since TC induces an overall crystalline phase in PF8, no changes in the linewidths till $6 \mathrm{GPa}$ indicate a lower compressibility perpendicular the chain axis. The sudden increase in the PL linewidths beyond 6 GPa implies a change in the 3-D crystalline phase. It is hard to predict exactly how the crystallinity changes beyond 6 GPa solely from optical measurements but these observations match with the low pressure coefficient of the PL energies at high pressures (Fig. 3).

\section{B. Pressure and temperature dependence of the Huang-Rhys factor}

The fine features in the absorption and emission spectra of conjugated molecules/polymers are described by Frank-Condon (FC) coupling. The $\pi-\pi *$ electronic transition is accompanied by a well resolved FC type progression of vibronic sub-bands. These vibronic bands are dominated by modes representing local C-C stretching in the vicinity of $1200-1600 \mathrm{~cm}^{-1}$. In the emission process, the Huang-Rhys factor $(S)$ 


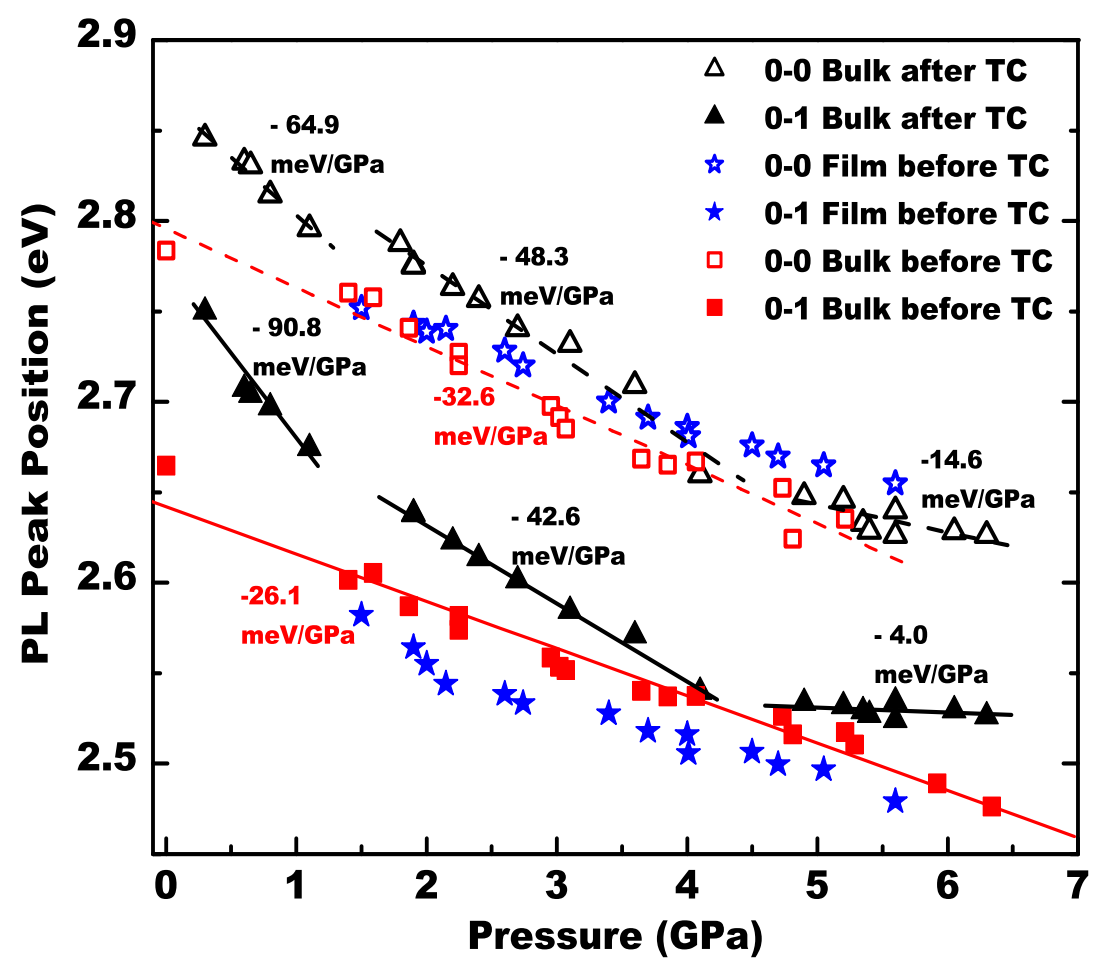

FIG. 3: Peak positions of the 0-0 and 0-1 PL vibronics of PF8 under pressure. The open and filled symbols represent the 0-0 and 0-1 peak positions, respectively. The blue symbols denote the thin film sample. The red and black symbols are from the bulk sample before and after TC, respectively.

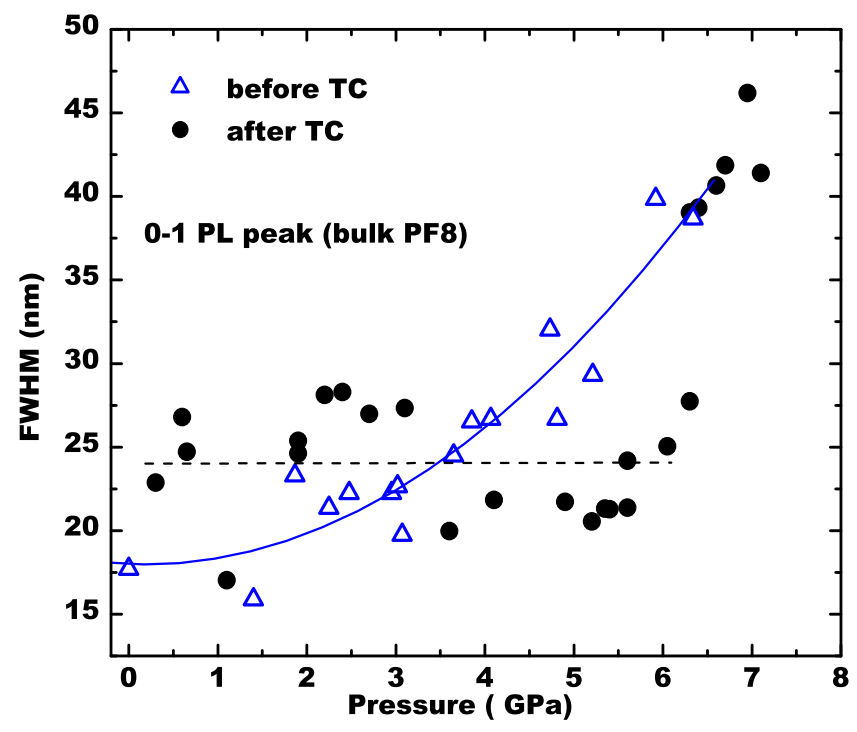

FIG. 4: FWHM (in nm) of the 0-1 PL vibronic as a function of pressure. The open $\triangle$ represents the as-is bulk sample and $\bullet$ represents the bulk sample after TC. 
corresponds to an average number of phonons that are involved when an excited molecule relaxes to its ground state configuration from its new equilibrium configuration in the excited state (after the absorption of a phonon). Assuming that the vibrational frequency is the same for ground and excited states and that the potentials are perfectly parabolic, $S$ may be experimentally determined from the fractional intensity of the vibronic peaks. The relative intensities of the features coupled by a single phonon frequency $(\omega)$ are described by

$$
\frac{I_{0 \rightarrow n}}{I_{\text {total }}}=\frac{e^{-S} S^{n}}{n !}
$$

where $I_{\text {total }}$ is the total intensity of individual transitions. $I_{0 \rightarrow n}$ is the intensity of the transition from the $0^{\text {th }}$ vibronic excited state to the $n^{\text {th }}$ vibronic state of the electronic ground state. The Huang-Rhys factor therefore corresponds to the average number of phonons that are involved when the excited molecule relaxes to its ground state configuration from the excited state, and $S \hbar \omega$ is the relaxation energy. $S$ may be determined from the fractional intensity of the vibronic peaks:

$$
S=\left(I_{0 \rightarrow 1}+2 I_{0 \rightarrow 2}+3 I_{0 \rightarrow 3}+\ldots\right) / I_{\text {total }} .
$$

$I_{0 \rightarrow 1}, I_{0 \rightarrow 2}$, and $I_{0 \rightarrow 3}$ refer to the intensity of the emission from the zeroth vibrational level excited state to the first, second, and third vibrational level of the ground state, respectively.

The relative strengths of the vibronic transitions change with both temperature and pressure. In a prior work we have shown that the Huang-Rhys factor in small molecules and long-chain polymers decreases with decreasing temperatures $\stackrel{10}{\underline{1}}$ The results for PF8 as a function of temperature are shown in Fig. 5, where the $S$-factor was determined by using Eq. (2) and the vibronic intensities beyond 0-3 were neglected. Smaller conjugated molecules typically show a larger value for $S$, which arises due to their large normal coordinate displacement between the ground and excited electronic states. The glassy phase in PF8 shows a much higher value for the Huang-Rhys factor compared to the $\beta$ phase, signaling planar conformations for the ground and excited states of the latter $\underline{32}^{2}$

At ambient pressure and RT the $S$-factor in PF8 is determined as 0.4 , similar to values obtained for the $\beta$-phase by Khan et al. $\frac{32}{2}$ Figure 5 plots the $S$-factor for PF8 both as function of temperature (at ambient pressure) and pressure (at RT) for a thin as-is film sample. At $40 \mathrm{~K}$ the $S$-factor is $\sim 0.25$ increasing to $\sim 0.4$ at RT. Upon increasing pressures, the $S$ - 


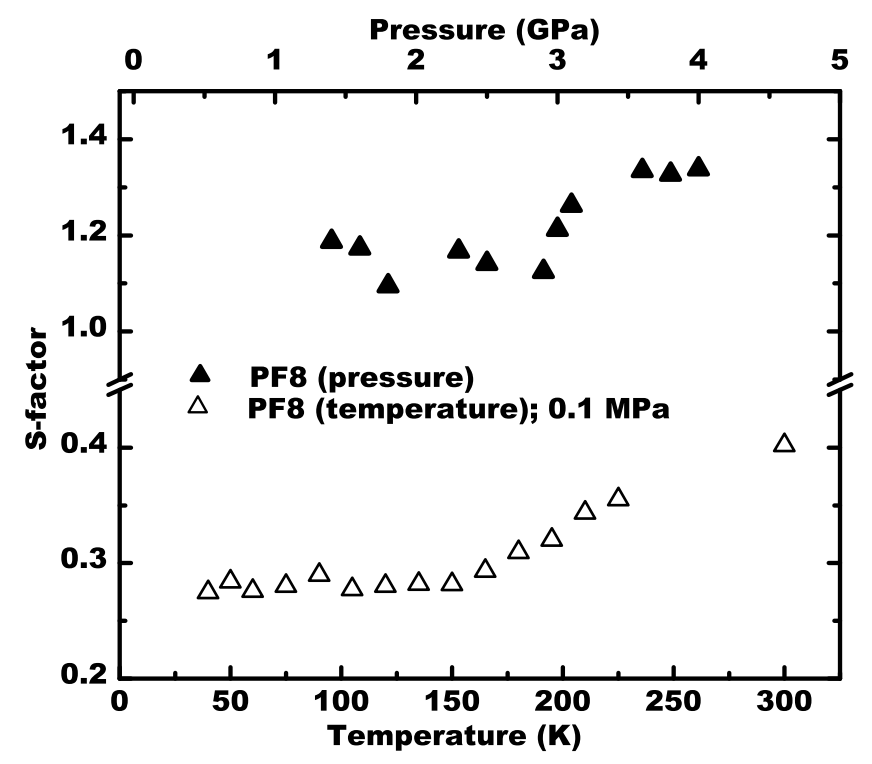

FIG. 5: The Huang-Rhys factor versus temperature and pressure in PF8.

factor is enhanced and almost remains a constant at 1.3, beyond 3.0 GPa. Lower values of the Huang-Rhys factor at ambient conditions imply a delocalized excited state along with a smaller geometric relaxation that follows a transition from the excited state to the ground state.

A comparison of the Huang-Rhys factors in PF8 as a function of temperature and pressure show that they increase, albeit at different rates. Upon increasing the temperature, the singlet excitons typically become more localized in smaller conjugated chain segments. $\underline{10}$ This localization results in a higher value for $S$ with increasing temperatures. The red-shift of the PL spectra upon increasing pressures is a clear sign of an enhanced effective conjugation and thus represents a higher delocalization of the excited state. This unambiguously shows that a higher value of the $S$-factor with enhanced pressures has a different origin as compared with enhanced temperatures; under enhanced pressures the geometric relaxation of the electronic states is increased, increasing the $S$-factor.

\section{RAMAN SCATTERING STUDIES}

The Raman spectrum of PFs is characterized by numerous intramolecular $\mathrm{C}-\mathrm{C} / \mathrm{C}-\mathrm{H}$ stretch and bend modes spanning from $100 \mathrm{~cm}^{-1}$ to $1600 \mathrm{~cm}^{-1}$. Both the vibrational frequencies and intensities determined by Raman spectroscopy are strongly influenced by variations in the backbone as well as side-chain conformations. The Raman peaks in the $1600 \mathrm{~cm}^{-1}$ 
region arise from an intra-ring $\mathrm{C}-\mathrm{C}$ stretch frequency and is best fit with two peaks: an overwhelmingly dominant peak at $1605 \mathrm{~cm}^{-1}$ and at least one or two weak peaks in the range of $1570-1600 \mathrm{~cm}^{-1}$. These weaker peaks correspond to a breathing motion of the pentagon within the monomer. The Raman frequencies in the $1250-1350 \mathrm{~cm}^{-1}$ region are associated with the backbone $\mathrm{C}-\mathrm{C}$ stretch motion. Due to the strong Raman peak of diamond, the $1200 \mathrm{~cm}^{-1}$ region is not observed in a DAC (Fig. 6 inset).

The low frequency Raman peaks in the $100-700 \mathrm{~cm}^{-1}$ range, shown in Figure 6 , originate from the alkyl side chains. This region is thus an extremely sensitive indicator of both side chain composition and ordering. The ring torsion mode at $480 \mathrm{~cm}^{-1}$ is seen in all PFs with various side group substitutions, and is almost independent of temperature. Since the Raman peaks around $600 \mathrm{~cm}^{-1}$ and in the 100-400 $\mathrm{cm}^{-1}$ range are mainly from alkyl side chains, they have very different signatures for various side chain substituted PFs. There are predominantly two Raman peaks in the $600 \mathrm{~cm}^{-1}$ region; the low frequency at $620 \mathrm{~cm}^{-1}$ corresponds to a stretching motion of the bridging $\mathrm{C}$ atom connected to the first $\mathrm{CH}_{2}$ group of the alkyl chain and the higher frequency peak at $633 \mathrm{~cm}^{-1}$ involves a torsional motion of the phenyl rings. Shorter alkyl chains such as ethyl-hexyl or an octyl group with gauche defects in PFs are characterized only by the higher frequency Raman peak in this region. The relative ratio of the 620 and $633 \mathrm{~cm}^{-1}$ Raman peaks in PF8 further track the presence of the $\mathrm{C}_{\beta}$ conformation. The intensity of the $633 \mathrm{~cm}^{-1}$ peak is stronger in the presence of the $\beta$ phase. It is clear from our ambient pressure data in Fig. 6 that the PF8 film had a significant fraction of $\mathrm{C}_{\beta}$ conformation before loading the DAC.

The $735 \mathrm{~cm}^{-1}$ peak in PFs correspond to the $747 \mathrm{~cm}^{-1}$ fundamental of a biphenyl with $\mathrm{A}_{1}$ symmetry. The stronger Raman peak in the $850 \mathrm{~cm}^{-1}$ region is a combination of ring distortion of the phenyl rings and a C-C stretch of the bridging carbon. The weaker Raman peaks around $900 \mathrm{~cm}^{-1}$ relate to C-C stretch type motion of the alkyl side chains. $\underline{\underline{15}}$ Frequencies in the 1100-1200 $\mathrm{cm}^{-1}$ region (not shown in Fig. 6) are sensitive to side-group substitution; the 1120 and $1135 \mathrm{~cm}^{-1}$ peaks mainly arise from the $\mathrm{C}-\mathrm{H}$ bending modes (either local or between phenyl units). 




FIG. 6: The Raman spectrum of as-is PF8 film at ambient pressure and RT at low frequencies. The doublet at $600 \mathrm{~cm}^{-1}$ marked by the arrows track the conformational isomers. The inset shows the high frequency region of the Raman spectrum of PF8 film; the sharp peak at $1320 \mathrm{~cm}^{-1}$ is the diamond peak from DAC.

\section{A. The $100-1200 \mathrm{~cm}^{-1}$ region}

Figure 7 shows the ambient pressure data of a PF8 film sample before and after TC along with the spectra of the bulk sample after TC under pressure in the low frequency region. Due to the red-shift of the PL spectrum under pressure, the background of the Raman spectrum is quite high in this region. We select two pressure points which are just above the discontinuity seen in the PL peak positions at $1.1 \mathrm{GPa}$. The ambient pressure results of PF8 after TC clearly shows the appearance of new peak at $370 \mathrm{~cm}^{-1}$. This is from the longitudinal accordion motion (LAM) of all anti conformation of the alkyl side chain. Such a conformation of the side chains preclude the $\beta$ phase suggesting that the individual backbone conformation is $\mathrm{C}_{\gamma}$ or $\mathrm{C}_{\alpha}$-type. The two pressure data upon $\mathrm{TC}$ show no signature of the $370 \mathrm{~cm}^{-1}$ peak, which means that the side chains at these pressures deviate from the all anti conformation, and the system is more like the $\mathrm{C}_{\beta}$ conformation. This correlates well with the PL data, where a different pressure coefficient is seen for the PL vibronics beyond 1.1 GPa, which we attribute to a more planar conformation of the backbone. We note that in the presence of the $\beta$ phase, LAM modes are not observed. Unfortunately, this low frequency region is difficult to systematically track in Raman scattering for all values of 


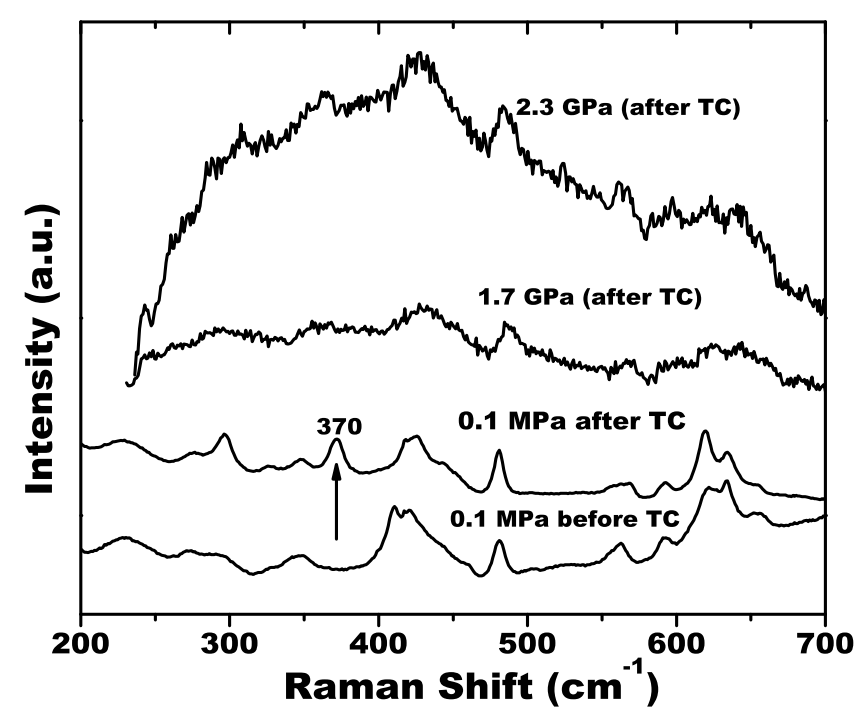

FIG. 7: Low frequency Raman spectra of PF8. The bottom two spectra are at ambient pressure before and after TC. The top two spectra are at elevated pressures after TC.

pressure due to the rising background.

The Raman peaks in the $1140 \mathrm{~cm}^{-1}$ region originate from a $\mathrm{CH}$ in-plane bend motion along with a ring distortion. In the planar $(\beta)$ conformation of the polymer or in a monomer there is mainly one Raman band at $1135 \mathrm{~cm}^{-1}$ that originates from the terminal phenyl rings. For nonplanar conformations, this motion splits as two or more vibrations originating from monomer units about the center of symmetry of the molecule and from the end rings. Upon thermal cycling the polymer from its $n$-LC phase, which concomitantly induces an overall crystalline phase and a reduction of the $\beta$ conformer, the 1135 and the $1172 \mathrm{~cm}^{-1}$ peaks broaden as seen in the ambient pressure data in Fig. 8 (a). Under enhanced pressures there is a broadening of the $1135 \mathrm{~cm}^{-1}$ peak; however, unlike ambient pressure, TC does not induce a further broadening of this peak at high pressures. The pressure coefficient of the $1135 \mathrm{~cm}^{-1}$ Raman peak is $1.6 \mathrm{~cm}^{-1} / \mathrm{GPa}$ and $2.9 \mathrm{~cm}^{-1} / \mathrm{GPa}$ for the as-is and thermallycycled samples, respectively. These pressure coefficients are lower compared to the high frequency $1600 \mathrm{~cm}^{-1}$ Raman peak, as shown in the next section.

\section{B. The $1600 \mathrm{~cm}^{-1}$ region}

A signature of the various conformational isomers is better deciphered by the backbone $\mathrm{C}-\mathrm{C}$ stretch modes in the $1300 \mathrm{~cm}^{-1}$ region. Unfortunately, under pressure in a DAC this 

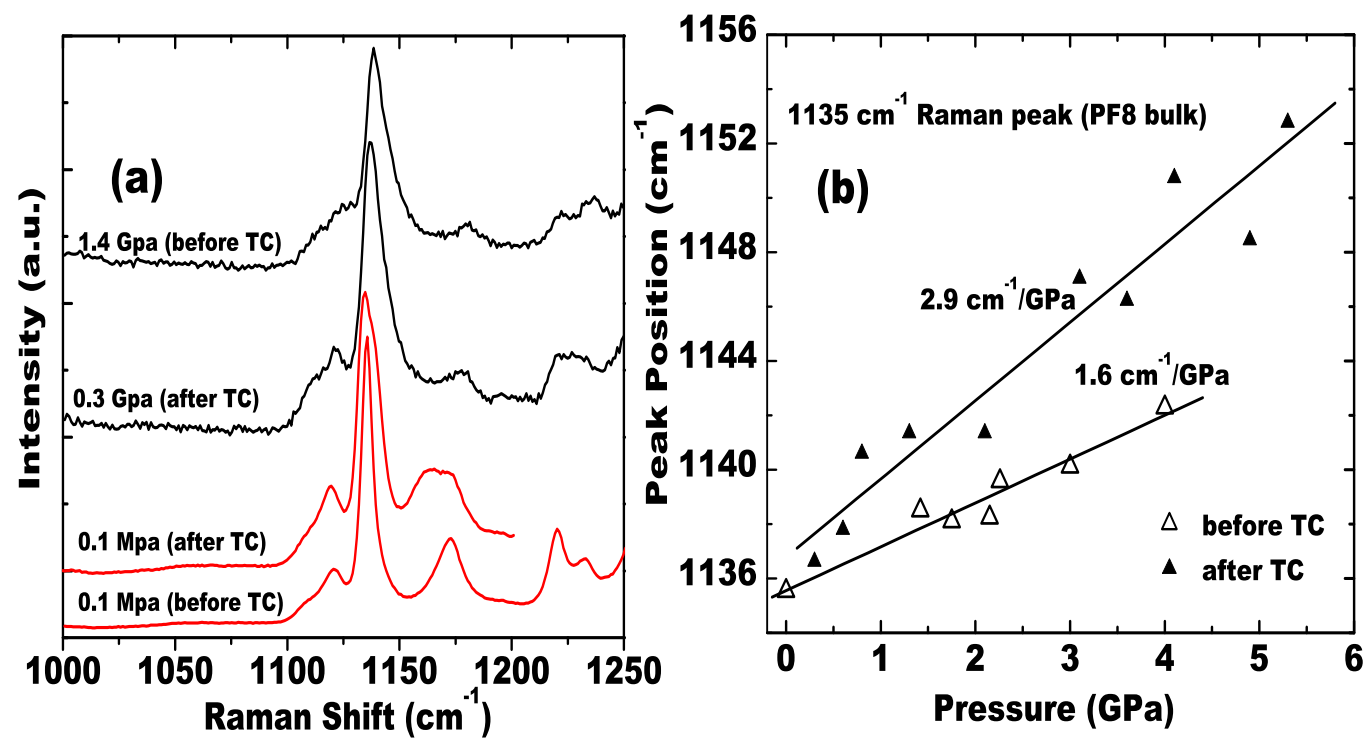

FIG. 8: (a) The Raman spectra in the $1100 \mathrm{~cm}^{-1}$ region at ambient pressure and higher pressures before and after TC. (b) Peak position of the $1135 \mathrm{~cm}^{-1}$ Raman peak before and after TC as a function of pressure.

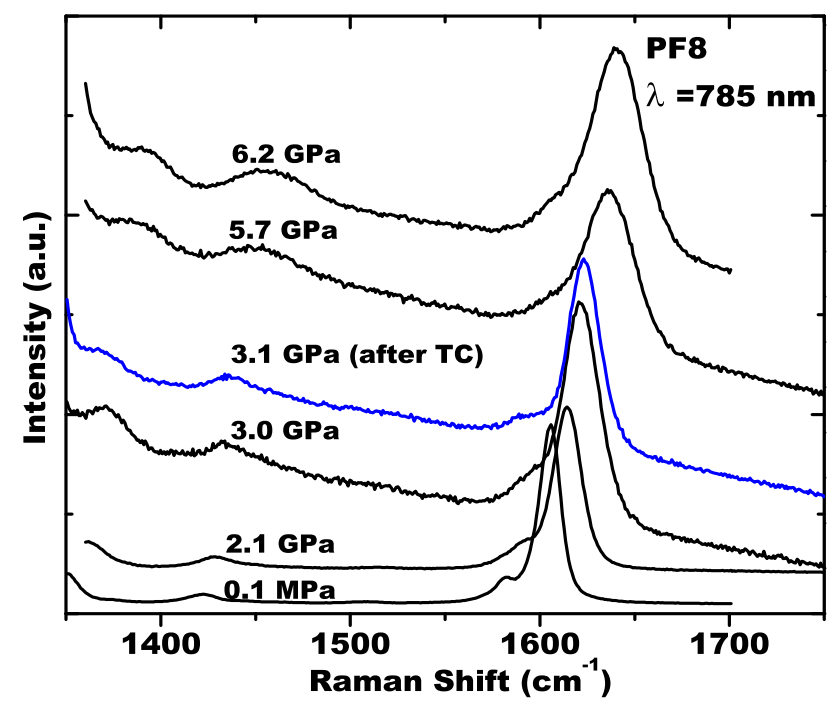

FIG. 9: Raman spectra of the $1600 \mathrm{~cm}^{-1}$ region (before TC) in $\mathrm{PF} 8$ at selected value of pressures. The spectrum at $3.1 \mathrm{GPa}$ is from the PF8 sample after TC.

region is swamped by the Raman peak of the diamond. The intra-ring C-C stretch peak at $1605 \mathrm{~cm}^{-1}$ is clearly seen at all pressures for both as-is and TC samples. We present a detailed analysis of this peak as a function of pressure in this section.

Since the origin of the $1600 \mathrm{~cm}^{-1}$ Raman peak is from intra ring C-C stretch motion, it is not sensitive to the various crystalline phases or conformations. Fig. 9 shows a few 
selected spectra of the $1600 \mathrm{~cm}^{-1}$ region of the as-is bulk sample under pressure; for a comparison the 3.1 GPa spectrum of the TC sample is also shown. Beyond 2.1 GPa the Raman background increases with a distinct asymmetry of the $1605 \mathrm{~cm}^{-1}$ peak, as shown in Fig. 9 . Such an asymmetry was observed in PF2/6 under pressure and was attributed to a strong electron-phonon interaction between the Raman peaks and the electronic continuum. ${ }^{27}$ This is characteristic of a Breit Wigner Fano (BFW) resonance; in PF2/6 many of the vibrational peaks also showed an anti-resonance behavior. To determine the peak position, asymmetry parameter, and linewidth as a function of pressure, we fit the $1605 \mathrm{~cm}^{-1}$ peak with a BWF line shape given by

$$
I(\omega)=I_{0} \frac{\left[\left(\omega-\omega_{0}\right) / q+\Gamma\right]^{2}}{\left(\omega-\omega_{0}\right)^{2}+\Gamma^{2}},
$$

where $\omega_{0}$ is the discrete phonon frequency, and $\Gamma$ is the width of the resonant interference between the continuum and discrete scattering channels. The asymmetry parameter $(1 / q)$ depends on the average electron-phonon matrix element, $M$, and the Raman matrix elements between the ground and excited states of the phonon and electron. The broadening parameter is given by $\Gamma=\pi M^{2} D(\omega)$, where $D(\omega)$ is the combined density of states for the electronic transitions. 35

Figure 10 (a) shows the peak position of the $1605 \mathrm{~cm}^{-1}$ Raman peak as a function of pressure. The pressure coefficient of the bulk PF8 sample before and after TC is almost the same at $7.2 \mathrm{~cm}^{-1} / \mathrm{GPa}$, which is higher than that observed for the $1135 \mathrm{~cm}^{-1}$ Raman peak. Pressure-induced changes in the vibrational frequencies is a measure of the Grunëisen parameter in the potential energy surface of the particular vibrational coordinate. For bulk inorganic solids the mode Grunëisen parameter is usually volume-independent. In molecular solids due to the differences between the local volume compression relative to that of the bulk, a volume dependent Grunëisen parameter is often observed. $\underline{\underline{36}}$ It is therefore not surprising that the pressure coefficient of the $1135 \mathrm{~cm}^{-1}$ and $1600 \mathrm{~cm}^{-1}$ Raman frequencies are different. We note that the mode Grunëisen parameter, which is given by $(1 / \omega) d \omega / d p$, is higher for the $1600 \mathrm{~cm}^{-1}$ peak compared to the $1135 \mathrm{~cm}^{-1}$ peak.

The inset of Fig. 10 (a) shows the linewidth increasing almost linearly with pressure for samples before and after TC. The assymetry parameter (1/q) from a BWF fit is small below 2.0 GPa, beyond which it increases rapidly with pressure as seen in Fig. 10 (b). Since the $1600 \mathrm{~cm}^{-1}$ peaks originate from an intra-ring $\mathrm{C}-\mathrm{C}$ stretch motion, thermal cycling and thus the changes in the induced phases have almost no impact on this Raman peak. The sign 



FIG. 10: (a) Position of the $1605 \mathrm{~cm}^{-1}$ Raman peak, obtained using a BWF fit, as a function of pressure for both as-is and TC PF8 bulk sample. The inset shows the linewidth of same peak as a function of pressure. (b) Asymmetry parameter (1/q) of the $1605 \mathrm{~cm}^{-1}$ Raman peak versus pressure. 1/q is obtained by fits to the Raman peak with a BWF line shape [Eq. (3).]

of the asymmetry parameter in a BWF resonance, which arises from an interaction of the electronic continuum, is an indicator of the energy of the continuum. The negative value of $\mathrm{q}$ in the fits show that the center of the electronic continuum lies below the discrete mode frequency of $1605 \mathrm{~cm}^{-1}(0.2 \mathrm{eV})$.

Since the vibrational frequencies of a harmonic solid are independent of compression, pressure induced changes in the Raman spectrum provide insight into the anharmonicity of the solid state potential. 37 The linear shift of the $1605 \mathrm{~cm}^{-1}$ Raman peak is a result of such an anharmonicity of the potential. Although one expects an additional shift of the phonon frequencies due to the BWF interaction, it is not possible to extract this information from our data due to the absence of a similar defect free polymer. The electronic continuum here arises from defect states such as the fluorenone defects or excimeric states. We see subtle differences between PF8 and PF2/6; the latter showed a higher asymmetry parameter and a square law dependence of the linewidth as a function of pressure. $\frac{27}{}$ The optical properties of PF8 and PF2/6 under pressure are further contrasted in the next section. 


\section{SUMMARY AND PROSPECT}

A comparison of the luminescence studies of as-is and thermally cycled PF8 under pressure shows many differences in the crystallinity and backbone conformations. Both samples show a red-shift of the PL energies upon increasing pressures but the TC sample shows three distinct region with phase transitions at $1.1 \mathrm{GPa}$ and $4.2 \mathrm{GPa}$. The $1.1 \mathrm{GPa}$ is attributed to a planarization between adjacent monomers where the $\mathrm{C}_{\alpha}$ and $\mathrm{C}_{\gamma}$ chormophores are driven towards the more planar $\mathrm{C}_{\beta}$ form. The as-is PF8 sample under pressure shows a similar behavior to as-is PF2/6 under pressure; the PL vibronics linearly shift with pressure although the nature of the emission at high pressures is quite different for the two. Beyond 4 GPa PF2/6 shows an orange emission compared to the greenish emission seen in PF8 at higher pressures. The orange emission in PF2/6 under pressure has been attributed to fluorenone defects and mixing of the singlet and charge-dipole states, which is enhanced due to the helicity of the backbone.

The linewidth of the PL vibronics as a function of pressure shows differences in the asis and TC PF8 samples. The as-is sample shows a broadening of the PL vibronics as a function of pressure. Such a dependence is seen in other amorphous polymers and signals an enhanced interchain interaction. The TC sample, which is in one of the crystalline phases, shows almost no change in the PL linewidths till $6 \mathrm{GPa}$. The main impact of pressure is to change the backbone conformation of the polymer once the polymer is thermally cycled. These results are similar to crystalline oligophenyls where there is hardly any change of PL linewidths with increasing pressures.

Similar to PF2/6, the Raman frequencies in PF8 harden with pressure. Due to limitations in a DAC only the $1135 \mathrm{~cm}^{-1}$ and the $1600 \mathrm{~cm}^{-1}$ Raman peaks could be tracked as a function of pressure. These intramolecular vibrations are not the best candidates for unravelling changes in the conformations or crystalline phases in PF8. The $1600 \mathrm{~cm}^{-1}$ shows asymmetric line shapes characteristic of a BWF resonance, signaling a strong electron-phonon interaction between the Raman phonons and the electronic continuum. The origin of the enhanced Raman linewdths is due to such electron-phonon interactions.

In light of our experimental results on the optical properties of PF8 under pressure, where a rich change in the phase morphology is observed, future XRD measurements under pressure would be invaluable to map these phase transitions in both PF8 and PF2/6. 


\section{Acknowledgments}

This work was partly supported through the National Science Foundation under grant No. ECCS-0523656. We thank M. Arif for the ambient pressure Raman data.

* Corresponding author E-mail:guhas@missouri.edu

1 M. Grell, D.D.C. Bradley, G. Ungar, J. Hill, and K.S. Whitehead, Macromolecules 32, 5810 (1999).

2 S. H. Chen, A. C. Su, C. H. Su, and S. A. Chen, Macromolecules 38, 379 (2005).

3 M. Knaapila, B.P. Lyons, T.P.A. Hase, C. Pearson, M.C. Petty, L. Bouchenoire, P. Thomspon, R. Serimaa, M. Torkkeli, and A.P. Monkman, Adv. Funct. Mater. 15, 1517 (2005).

4 B. Tanto, S. Guha, C.M. Martin, U. Scherf, and M.J. Winokur, Macromolecules 37, 9438 (2004).

5 M.H. Rahman, C-Y. Chen, S-C. Liao, H-L. Chen, C-S. Tsao, J-H. Chen, J-L. Liao, V.A. Ivanov, and S-A. Chen, Macromolecules 406572 (2007).

6 M. Knaapila, V.M. Garamus, F.B. Dias, L. Almasy, F. Galbrecht, A. Charas, J. Morgado, H.D. Burrows, U. Scherf, and A.P. Monkman, Macromolecules 39, 6505 (2006).

7 A.J. Cadby, P.A. Lane, H. Mellor, S.J. Martin, M. Grell, C. Giebeler, D.D.C. Bradley, M. Wohlgenannt, C. An, and Z.V. Vardeny, Phys. Rev. B 62, 15604 (2000).

8 M.J. Winokur, J. Slinker, and D.L. Huber, Phys. Rev. B 67, 184106 (2003).

9 H. Liem, P. Etchegoin, K.S. Whitehead, and D.D.C. Bradley, J. Appl. Phys. 92, 1154 (2002).

10 S. Guha, J. D. Rice, Y. T. Yau, C. M. Martin, M. Chandrasekhar, H.R. Chandrasekhar, R. Guentner, P. S. de Freitas, and U. Scherf, Phys. Rev. B 67, 125204 (2003).

11 G. Lieser, M. Oda, T. Miteva, A. Meisel, H.G. Nothofer, and U. Scherf, Macromolecules 33, $4490(2000)$.

12 W. Chunwaschirasiri, B. Tanto, D. L. Huber, and M.J. Winokur, Phys. Rev. Lett. 94, 107402 (2005).

13 S.H. Chen, H.L. Chou, A.C. Su, and S. Chen, Macromolecules 376833 (2004).

14 M. Arif, C. Volz, and S. Guha, Phys. Rev. Lett. 96, 025503 (2006).

15 C. Volz, M. Arif, and S. Guha, J. Chem. Phys. 126, 064905 (2007).

16 W. C. Tsoi and D. G. Lidzey, J. Phys.: Condens. Matter 20, 125213 (2008). 
17 S. Guha, W. Graupner, S. Yang, M. Chandrasekhar, and H.R. Chandrasekhar, Approaches to Polar Order, Ed. R. Glaser and P. Kaszynski, ACS Symposium Series 798, 127 (2001).

18 V. R. Gangilenka, A. DeSilva, H. P. Wagner, R. E. Tallman, B. A. Weinstein, and R. Scholz, Phys. Rev. B 77, 115206 (2008).

19 M. Chandrasekhar, S. Guha, and W. Graupner, Adv. Mater. 13, 613 (2001).

20 S. Webster and D.N. Batchelder, Polymer 37, 4961 (1996).

21 G. Yang, Y. Li, J.O. White, and H.G. Drickamer, J. Phys. Chem. B 103, 7853 (1999).

22 V. Morandi, M. Galli, F. Marabelli, and D. Comoretto, Phys. Rev. B 79045202 (2009).

23 J.P. Schmidtke, R. H. Friend, and C. Silva, Phys. Rev. Lett. 100, 157401 (2008).

24 S.c. Yang, W. Graupner, S. Guha, P. Puschnig, C. Martin, H. R. Chandrasekhar, M. Chandrasekhar, G. Leising, C. Ambrosch-Draxl, Phys. Rev. Lett. 85, 2388 (2000).

25 S. Guha, W. Graupner, R. Resel, M. Chandrasekhar, H.R. Chandrasekhar, R. Glaser, and G. Leising, Phys. Rev. Lett. 82, 3625 (1999).

26 C.M. Martin, Q. Cai , S. Guha, W. Graupner, M. Chandrasekhar, and H.R. Chandrasekhar, Physica Status Solidi (b) 241, 3339 (2004).

27 C.M. Martin, S. Guha, M. Chandrasekhar, H.R. Chandrasekhar, R. Guentner, P. Scanduicci de Freitas, U. Scherf, Phys. Rev. B 68, 115203 (2003).

28 H. Cheun, B. Tanto, W. Chunwaschirasiri, B. Larson, and M.J. Winokur, Appl. Phys. Lett 84, $22(2004)$.

29 G. Heimel, K. Hummer, C. Ambrosch-Draxl, W. Chunwachirasiri, M. J. Winokur, M. Hanfland, M. Oehzelt, A. Aichholzer, and Roland Resel, Phys. Rev. B 73, 024109 (2006)

30 H. G. Drickamer and C. W. Frank, Electronic Transitions and the High Pressure Chemistry and Physics of Solids, (Chapman and Hall, Ltd., 1973).

31 C. Ambrosch-Draxl, J. A. Majewski, P. Vogl, and G. Leising, Phys. Rev. B 51, 9668 (1995).

32 A. L. T. Khan, P. Sreearunothai, L. M. Herz, M. J. Banach, and A. Köhler, Phys. Rev. B 69, 085201 (2004).

33 M. Pope and C.E. Swenberg, Electronic Processes in Organic Crystals and Polymer, (Oxford University Press, Oxford 1999).

34 M. Knaapila, R. Stepanyan, B. P. Lyons, M. Torkkeli, T.P.A. Hase, R. Serimaa, R. Güntner, O.H. Seeck U. Scherf, and A. P. Monkman, Macromolecules 382744 (2005).

35 M.V. Klein, Light Scattering in Solids, edited by M. Cardona (Springer-Verlag, New York, 
1975).

36 R. Zallen and M. L. Slade, Phys. Rev. B 18, 5775 (1978).

37 J.R. Ferraro, Vibrational spectroscopy at high external pressures, Academic Press, New York (1984). 